\title{
UPPER SEMICONTINUITY PROPERTIES OF SET VALUED FUNCTIONS
}

\author{
L. Cesari and M. B. Suryanarayana \\ Department of Mathematics, University of Michigan, Ann Arbor, MI 48104, U.S.A.
}

(Received 16 November 1978)

Key words: Lower closure, upper semicontinuity, set valued functions, conjugate duality, seminormality. Lipschitz type conditions.

\section{INTRODUCTION}

IN THE present paper we intend to present lower closure theorems in their generality and to discuss their relevant hypotheses, particularly the role of upper semicontinuity properties of the relevant sets, in the light of recent work of the authors, and of Goodman, Olech, Ioffe and Rockafellar.

The uppersemicontinuity property (Q) for closed convex set valued functions $y \rightarrow Q(y) \subset B$ (Cesari $[1,2]$ ) is usually expressed in terms of union, intersections and closures on subsets $Q(y)$ of a Banach space $B$ depending on an index $y$ ranging on a subset $A$ of a metric space $(Y, d)$ :

$$
Q\left(y_{0}\right)=\cap_{\delta>0} \operatorname{cl} \operatorname{co} \cup\left[Q(y), d\left(y, y_{0}\right) \leqslant \delta\right] .
$$

The analogous upper semicontinuity property $(\mathrm{K})$ for closed set valued functions, or Kuratowski's condition, is then expressed by the requirement

$$
Q\left(y_{0}\right)=\cap_{\delta>0} \mathrm{cl} \cup\left[Q(y), d\left(y, y_{0}\right) \leqslant \delta\right] .
$$

In Section 2 we present lower closure theorems in Banach spaces including some which we are using elsewhere for Pareto problems in Banach space situations where the relevant functional has values in such a space with ordering defined by convex cones.

In Section 3 we show that whenever the sets $Q$ are in a finite dimensional space, lesser requirements are needed in the lower closure theorems. Indeed, in such situations we had already shown in 1968 [2] that suitable growth properties imply property (Q). This remark can now be used to prove property $(Q)$ for certain auxiliary sets which can be made to play a role in the proofs of the lower closure theorems. Actually property $(Q)$ in one form or another is indeed used in the proofs of lower closure theorems, implicitly or explicitly.

In Section 4 we summarize recent relevant results of Goodman [3] which prove that the same property (Q) can be expressed in terms of duality operations of convex analysis, and thus showing the equivalence of property $(Q)$ with properties used by Ioffe [4], Olech [5, 6] and Rockafellar [7] in their lower closure theorems. Thus, our lower closure theorems (Section 2), covering Banach space situations, with ordering defined by convex cones, appear to be more general than those of the aforementioned authors. We are indeed using them in Pareto problems [8,9] at such level of generality.

In Section 5, again in Banach space situation, we show that property (Q) can be expressed in terms of seminormality conditions, in the lines, that is, of Tonelli's early normality condition and as later used by McShane. (See [10-12] for an analogous discussion in finite dimensional spaces.) 
In Section 7 we show that, even in Banach space situations, natural blendings of weak Lipschitz and other hypotheses imply property (Q), and this leads to the formulation of a series of simple criteria of wide applicability, particularly in Banach space situations where growth conditions alone may not suffice. We state in Section 7 an analytic condition, or property (D) (cf [14]) which includes the above mentioned criteria, which implies a weak form of property (Q)-and this in turn implies lower closure even in Banach space situations.

Thus, for instance, a weak Lipschitz assumption as

$$
\left\|f\left(t, y_{1}, u(t)\right)-f\left(t, y_{2}, u(t)\right)\right\| \leqslant F(t, u(t))\left\|y_{1}-y_{2}\right\|
$$

together with the boundedness assumption

$$
\int_{t_{t}}^{t_{2}} F(t, u(t)) \mathrm{d} t \leqslant M
$$

imply property $(Q)$. This remark, which had been made already in $[9,13,14]$, seems to have been missed by both R. Berkovitz and G. R. Bates in their recent presentations (see Section 7 and $[19,20])$.

\section{STATEMENT OF LOWER CLOSURE THEOREMS}

Part (a): Orientor field formulation.

Here $(G, \alpha, \mu)$ will denote a finite complete measure space of elements $t$ with a finite measure $\mu$ and a $\sigma$-algebra $\alpha$ of $\mu$-measurable subsets. Also, $(G, \rho)$ is a metric space with a distance function $\rho$. Besides $(Y, d)$ is a metric space of elements $y$ with the distance function $d$. Let $Z$ and $B$ be Banach spaces over the reals $\mathbf{R}$, and let us assume that a nonempty convex closed cone $\Lambda$ is assigned in $Z$.

Let $Z^{*}$ and $B^{*}$ denote the duals of the spaces $Z$ and $B$ and let $\left\langle\eta^{*}, \eta\right\rangle,\langle\zeta, \xi\rangle$ denote the linear operations $\eta^{*} \in Z^{*}, \zeta \in B^{*}$ applied to the elements $\eta \in Z, \xi \in B$. Let $\Lambda^{*}$ denote the polar of the cone $\Lambda$, that is, the set of all $\eta^{*} \in Z^{*}$ such that $\left\langle\eta^{*}, \eta\right\rangle \leqslant 0$ for all $\eta \in \Lambda$. We shall assume that $\Lambda$ has the angle property, that is, there is a number $\varepsilon \in \mathbf{R}, 0<\varepsilon \leqslant 1$, and an element $\zeta \in-\Lambda^{*}$, $\zeta \neq 0$, such that $\Lambda \subset[\eta \in Z,\langle\zeta, \eta\rangle \geqslant \varepsilon\|\zeta\|\|\eta\|]$. Let $A$ denote a given subset of $G \times Y$ and let $A(t)$ be the set $A(t)=[y \in Y \mid(t, y) \in A]$, that is, the section of $A$. We shall assume that $A(t) \neq \varnothing$ for all $t \in G$.

First we state lower closure theorems for problems of optimal control (including Pareto problems) when the control parameters are eliminated and the problems are expressed in terms of orientor fields.

(2.i) (A lower closure theorem in Banach spaces in terms of orientor fields).

Let $(G, \rho),(Y, d)$ be metric spaces of which $G$ is also a finite complete measure space $(G, \alpha, \mu)$. Let $Z, B$ be Banach spaccs over the reals $\mathbf{R}$, and let us assume that $Z$ is reflexive and that in $Z$ a convex closed cone $\Lambda$ has been assigned with the angle property. Let $A$ be any subset of $G \times Y$ such that the sections $A(t), \quad t \in G$, are all nonempty, and also closed in $(Y, d)$ for $\mu$-almost all $t \in G$. Let $\bar{Q}(t, y), y \in A(t), t \in G$, be given nonempty subsets of $Z \times B$. Let $\xi(t), y(t), \eta_{k}(t), \xi_{k}(t), y_{k}(t), \varphi(t)$, $t \in G, k=1,2, \ldots$, be given $\mu$-measurable functions with $\xi, \xi_{k} \in L_{1}(G, B)$ and $\eta_{k}, \varphi \in L_{1}(G, Z)$. Let us assume that

(2.1) for $\mu$-almost all $t \in G$, the sets $\widetilde{Q}(t, y), y \in A(t)$, are closed, convex and satisfy property (Q) with respect to $y$ along the trajectory $y(t)$, that is, for $y=y(t)$. 
(2.2) $y(t), y_{k}(t) \in A(t),\left(\eta_{k}(t), \xi_{k}(t)\right) \in \widetilde{Q}\left(t, y_{k}(t)\right), t \in G(\mu$-a.e. $), k=1,2, \ldots$,

(2.3) - $-\infty<i=\lim _{\inf _{k \rightarrow \infty}} \int_{G}\left\langle\zeta, \eta_{k}(t)\right\rangle \mathrm{d} \mu<+\infty$, where $\zeta \in Z^{*}$ is the element mentioned in the
angle property, (2.4) $\xi_{k} \rightarrow \xi$ weakly in $L_{1}(G, B), y_{k}(t) \rightarrow y(t)$ in measure in $G$ as $k \rightarrow \infty$,

(2.5) $\eta_{k}(t)-\phi(t) \in \Lambda, t \in G,(\mu$-a.e.).

Then there is a function $\eta(t), t \in G, \eta \in L_{1}(G, Z)$, such that

$$
(\eta(t), \xi(t)) \in \widetilde{Q}(t, y(t)),(\mu \text {-a.e. }), \text { and } \int_{G}\langle\zeta, \eta(t)\rangle \mathrm{d} \mu \leqslant i .
$$

Remark 1. Note that in (2.1) we require that for every $\bar{t} \in G$ we have

$$
\widetilde{Q}(\bar{t}, y(\bar{t}))=\cap_{\delta>0} \operatorname{cl} \operatorname{co} \cup\left[\widetilde{Q}(\bar{t}, y), d\left(y, y_{0}\right)<\delta\right], \tilde{t} \in G .
$$

Actually, all that is needed here is that for a suitable sequence $k_{s}$ we have

$$
Q(\bar{t}, y,(\bar{t})) \supset \cap_{h=1}^{\infty} \operatorname{cl} \operatorname{co}\left[\cup_{s=h}^{\infty} \tilde{Q}\left(\bar{t}, y_{k_{s}}(\bar{t})\right)\right], \tilde{t} \in G(\mu-\text { a.e. }) .
$$

This is a form of property (Q) with respect to $y$ only, with respect to a given sequence, and only at the points $(t, y(t))$, that is, along the trajectory.

Remark 2. Note that, instead of (2.5) we could require only that $(2.5)^{\prime}$ there are real valued functions $\lambda(t), \lambda_{k}(t), t \in G, \lambda, \lambda_{k} \in L_{1}(G, R), k=1,2, \ldots$, such that $\left(\zeta, \eta_{k}(t)\right\rangle \geqslant \lambda_{k}(t), t \in G, k=1,2, \ldots$, and $\lambda_{k} \rightarrow \lambda$ weakly in $L_{1}(G . R)$.

(2.ii) As in (2.i) with $Z=\mathbf{R}^{v}, B=\mathbf{R}^{n}$ and property (K) replacing property (Q) in (2.1). Then the same conclusion as in (2.i) holds.

(2.iii) As in (2.i) with $Z$ and $B$ Banach spaces, with $\xi_{k} \rightarrow \xi$ strongly in $L_{1}(G, B)$ and property (K) replacing property $(Q)$ in (2.1). Then the same conclusion as in (2.i) holds.

For $B$ and $Z$ general Banach spaces the following also holds:

(2.iv) As in (2.i) with $Z$ and $B$ Banach spaces as stated, with (2.1) replaced by $(2.1)^{\prime}$ : for almost all $t \in G$, the set $\widetilde{Q}(t, y(t))$ is closed and convex. Moreover, we consider here functions; $\xi(t), y(t), \eta_{k}(t)$, $\bar{\eta}_{k}(t), \xi_{k}(t), \bar{\xi}_{k}(t), y_{k}(t), \phi(t), t \in G=1,2, \ldots$ all $\mu$-measurable in $G$, such that $\xi, \xi_{k}, \bar{\xi}_{k} \in L_{1}(G, B)$, $\eta_{k}, \bar{\eta}_{k}, \phi \in L_{1}(G, Z)$ satisfy $(2.3)$ and also

$(2.2)^{\prime}: y(t), y_{k}(t) \in A(t),\left(\eta_{k}(t), \xi_{k}(t)\right) \in \widetilde{Q}\left(t, y_{k}(t)\right),\left(\bar{\eta}_{k}(t), \bar{\xi}_{k}(t)\right) \in \widetilde{Q}(t, y(t)), t \in G(\mu$-a.e. $), k=1,2, \ldots$

$(2.4)^{\prime}: \xi_{k} \rightarrow \xi$ weakly in $L_{1}(G, B)$ as $k \rightarrow \infty$ and Property (D):

$$
\delta_{k}^{0}(t)=\eta_{k}(t)-\bar{\eta}_{k}(t) \rightarrow 0, \delta_{k}(t)=\xi_{k}(t)-\bar{\xi}_{k}(t) \rightarrow 0
$$

in measure in $G$ as $k \rightarrow \infty$. Then, the same conclusion as in (2.i) holds.

Theorems (2.i-iv) were essentially proved in various stages, by Cesari $[12,15]$, Cesari and Suryanarayana $[8,9,14]$ and Kaiser and Suryanarayana [16]. Thus, we see from (2.ii) that 
property $(\mathrm{Q})$ can be disregarded if property $(\mathrm{K})$ holds and spaces $Z$ and $B$ are finite dimensional; we see from (2.iv) that both property $(Q)$ and property $(K)$ can be disregarded if the analytic property (D) holds for Banach spaces $Z$ and $B$ as stated. However, as already mentioned, the hypotheses of Theorem (2.ii), particularly the weak convergence $\xi_{k} \rightarrow \xi$, imply that certain auxiliary sets $Q^{*}(t, y)$, essential in the proofs, have property (Q) with respect to $y$. Concerning Theorem (2.iv), property (D) itself implies a certain weak form of property (Q) for the relevant sets, which is strong enough for the entire argument based on this property to hold (see Section 7 of this paper).

Part (b): Optimal control formulation.

We now present statements, analogous to the previous ones, when we consider problems of optimal control in their usual form involving controls:

Let $(G, \rho),(G, \alpha, \mu),(Y, d), Z, B$ be spaces as in (a) and let $U$ be a further Banach space over the reals. Let $A$ and $A(t), \quad t \in G$, as in (2.i) and for every $(t, y) \in A$ let $U(t, y)$ be a given subset of $U$. Let $M$ denote the set $\{(t, y, u) \mid(t, y) \in A, u \in U(t, y)\}$ and let $f_{0}(t, y, u), f(t, y, u)$ be functions defined on $M$ with values in $Z$ and $B$ respectively, for which we assume the following condition replacing measurability hypotheses:

Property (C). For each $\varepsilon>0$, there is a compact subset $K$ of $G$ with $\mu(G-K)<\varepsilon$ such that the sets $A_{K}=\{(t, y) \in A \mid t \in K\}$ and $M_{K}=\{(t, y, u) \in M \mid t \in K\}$ are closed and the restrictions to $M_{K}$ of $f$ and $f_{0}$ are continuous.

An alternate condition, instead of (C), is as follows.

Property $\left(\mathrm{C}^{\prime}\right)$. The spaces $G, Y, Z, B, U$ as above are separable, $A=G \times A_{0}, A_{0} \subset Y, \quad U(t, y)$ $=U_{0} \subset U, \quad A_{0}, U_{0}$ fixed sets, $A_{0}$ closed in $Y$, the functions $f_{0}(t, y, u), f(t, y, u)$ defined in $G \times A_{0} \times U_{0}$ are $\mu$-measurable in $t$ for every $(y, u) \in A_{0} \times U_{0}$ and continuous in $(y, u)$ for $\mu$-almost all $t \in G$.

For every $(t, y) \in A$ let us denote by $\widetilde{Q}(t, y)$ the set

$$
\widetilde{Q}(t, y)=\left\{(\eta, \xi) \mid \eta \in f_{0}(t, y, u)+\Lambda, \xi=f(t, y, u), u \in U(t, y)\right\} \subset Z \times B .
$$

(2.v) (A lower closure theorem in Banach spaces in terms of control parameters).

Let us assume that (H1) for $\mu$-almost all $t \in G$ the sets $\tilde{Q}(t, y), \quad y \in A(t)$, satisfy property (Q) with respect to $y$ only, at $y=y(t)$ in $A(t)$. Let $\xi(t), y(t), \xi_{k}(t), \eta_{k}(t), y_{k}(t), u_{k}(t), \phi(t), t \in G, k=1,2, \ldots$, be $\mu$-measurable functions, $\xi, \xi_{k} \in L_{1}(G, B), \quad \eta_{k}, \phi \in L_{1}(G, Z)$, such that

$$
\begin{array}{r}
y(t), y_{k}(t) \in A(t) \subset Y, \quad u_{k}(t) \in U\left(t, y_{k}(t)\right) \subset U, \quad \eta_{k}(t)-\phi(t) \in \Lambda, \quad t \in G, \\
\eta_{k}(t) \in f_{0}\left(t, y_{k}(t), u_{k}(t)\right)+\Lambda, \quad \xi_{k}(t)=f\left(t, y_{k}(t), u_{k}(t)\right), \quad t \in G, \quad \mu \text {-a.e., }
\end{array}
$$

(2.8) $\xi_{k} \rightarrow \xi$ weakly in $L_{1}(G . B), \quad y_{k}(t) \rightarrow y(t)$ in measure in $G$ as $k \rightarrow \infty$. 
Then, there are functions $\eta(t), t \in G, \quad \eta \in L_{1}(G, Z)$ and $u(t), \quad t \in G, \mu$-measurable in $G$, such that

$$
\eta(t) \in f_{0}(t, y(t), u(t))+\Lambda, \quad \xi(t)=f(t, y(t), u(t)), \quad u(t) \in U(t, y(t)) \subset U, t \in G(\mu \text {-a.e. })
$$

and

$$
-\infty<\int_{G}\langle\zeta, \eta(t)\rangle \mathrm{d} \mu \leqslant i
$$

A remark similar to Remark 2 after (2.i) holds also here.

(2.vi) As in (2.v) with $Z=\mathbf{R}^{v}, B=\mathbf{R}^{n}$ and property (K) replacing property (Q) in (2.v). Then the same conclusion as in (2.v) holds.

(2.vii) As in (2.v) with $Z$ and $B$ Banach spaces as stated, with $\xi_{k} \rightarrow \xi$ strongly in $L_{1}(G, B)$ and property $(K)$ replacing property $(Q)$ in $(2 . v)$. Then the same conclusion as in $(2 . v)$ holds.

(2.viii) As in (2.v) with $Z$ and $B$ Banach spaces as stated, with $U(t) \subset U$ depending on $t$ only, and (II1) replaced by (H1)': for $\mu$-almost all $t \in G$, the set $\widetilde{Q}(t, y(t))$ is closed and convex. Moreover, we assume that functions $\xi(t), y(t), \eta_{k}(t), \bar{\eta}_{k}(t), \xi_{k}(t), \xi_{k}(t), y_{k}(t), u_{k}(t), \phi(t), t \in G, k=1,2, \ldots$, are assigned, $\xi, \xi_{k}, \bar{\xi}_{k} \in L_{1}(G, B), \quad \eta_{k}, \eta_{k}, \phi \in L_{1}(G, Z)$, satisfying (2.7) and also,

$(2.6)^{\prime} y_{k}(t) \in A(t), \quad \eta_{k}(t) \in f_{0}\left(t, y_{k}(t), u_{k}(t)\right)+\Lambda$

$\xi_{k}(t)=f\left(t, y_{k}(t), u_{k}(t)\right), \quad u_{k}(t) \in U(t)$,

$\bar{\zeta}_{k}(t)=f\left(t, y(t), u_{k}(t)\right), \quad \bar{\eta}_{k}(t) \in f_{0}\left(t, y(t), u_{k}(t)\right)+\Lambda$,

$(2.8)^{\prime} \xi_{k} \rightarrow \xi$ weakly in $L_{1}(G, B), \quad y_{k}(t) \rightarrow y(t)$ in measure in $G$ as $k \rightarrow \infty$, and

(D)

$$
\delta_{k}(t)=f\left(t, y_{k}(t), u_{k}(t)\right)-f\left(t, y(t), u_{k}(t)\right) \rightarrow 0,
$$

$$
\delta_{k}^{0}(t)=f_{0}\left(t, y_{k}(t), u_{k}(t)\right)-f_{0}\left(t, y(t), u_{k}(t)\right) \rightarrow 0
$$

in measure in $G$ as $k \rightarrow \infty$. Then, the same conclusion as in (2.v) holds.

The above versions of the lower closure theorems are essentially given in the same papers $[8,9,12,14-16]$ mentioned above. Also, in [14], great many simple easily verifiable explicit conditions are given which guarantee that condition (D) holds, some of which are reported in Section 7 below in the Banach space situation.

For the classical integrals of the calculus of variations

$$
I[y]=\int_{a}^{b} f_{0}\left(t, y(t), y^{\prime}(t)\right) \mathrm{d} t, \quad-\infty<a<b<+\infty,
$$

$f_{0}(t, y, z)$ continuous in $[a, b] \times R^{2}, y(t)$ absolutely continuous in $[a, b]$, the convexity of $f_{0}(t, y, z)$ with respect to $z$ is a necessary and sufficient condition for the lower semicontinuity of $I[y]$ with respect to the usual weak topology $y_{k} \rightarrow y$ uniformly, $y_{k}^{\prime} \rightarrow y^{\prime}$ weakly in $L_{1}([a, b], R)$ (Cesari [21]).

For the general situation depicted under (2.ii) and (2.vi) and variants (see, e.g., Remark 2), and corresponding necessary and sufficient conditions, we refer to Ioffe [4] and Cesari [17]. 


\section{GROWTH PROPERTIES AND UPPER SEMICONTINUITY PROPERTY (Q) OF SET VALUED FUNCTIONS}

Part (a): Orientor field formulation.

Let $B$ and $Z$ denote Banach spaces over the reals, and $(Y, d)$ be a metric space. Let $\mathbf{R}^{n}$ denote the $n$-dimensional Euclidean space. Let $A$ be any subset of $Y$ and for each $y \in A$ let $Q(y)$ be a given nonempty subset of $Z \times B$. The following three uppersemicontinuity properties are relevant to us.

We say that $Q(y), y \in A$, is uppersemicontinuous by set inclusion at $y_{0} \in A$ provided, given $\varepsilon>0$, there is $\delta>0$ such that $d\left(y, y_{0}\right)<\delta$ implies $Q(y) \subset\left[Q\left(y_{0}\right)\right]_{\varepsilon}$, where $Q\left(y_{0}\right)_{c}$ denotes the $\varepsilon$-neighborhood of $Q\left(y_{0}\right)$ in $Z \times B$, and $N_{\delta}\left(y_{0}\right)$ denotes the $\delta$-neighborhood of $y_{0}$ in $A$.

$$
Q\left(y_{0}\right)=\cap_{\delta>0} \mathrm{cl} \cup\left\{Q(y), d\left(y, y_{0}\right)<\delta\right\}
$$

where cl denotes weak closure in $B$.

We say, as in $[1,2]$ and in Section 1 , that $Q(y), y \in A$, satisfies the uppersemicontinuity property $(\mathrm{Q})$ at $y_{0} \in A$ provided

$$
Q\left(y_{0}\right)=\cap_{\delta>0} \operatorname{clco} \cup\left\{Q(y), d\left(y, y_{0}\right)<\delta\right\} .
$$

Here cl operates on convex sets and thus weak and strong closures coincide.

We may say that $Q(y)$ has any of these properties in $A$ if the same property holds at every $y_{0} \in A$. Obviously, if $Q(y)$ has property $(\mathrm{K})$ at $y_{0}$ then $Q\left(y_{0}\right)$ is closed; if $Q(y)$ has property $(\mathrm{Q})$ at $y_{0}$ then $Q\left(y_{0}\right)$ is closed and convex. Moreover, property (Q) implies property $(\mathrm{K})$.

If $A$ is closed in $Y$, then the sets $Q(y), y \in A$, have property $(\mathrm{K})$ in $A$ if and only if the graph of $Q(y)$, or $[(y, \xi) \mid y \in A, \xi \in Q(y)]$ is closed in $Y \times(Z \times B)[1]$.

Moreover, if the set valued function $Q(y)$ is uppersemicontinuous at $y_{0} \in A$ by set inclusion, and $Q\left(y_{0}\right)$ is closed, then $Q(y)$ has property $(\mathrm{K})$ at $y_{0}$; if $Q\left(y_{0}\right)$ is also convex and closed, then $Q(y)$ has property $(\mathrm{Q})$ at $y_{0}$. In [16] an "intermediate" property $\left(\mathrm{Q}^{\prime}\right)$ was introduced (intermediate between $(K)$ and $(Q)$ properties), and these statements were extended there to property $\left(Q^{\prime}\right)$.

Also, in [2] Cesari proved that property (K) for certain convex sets which are "bounded below" guarantees property (Q) for certain associated auxiliary sets $Q^{*}(y)$, which are relevant in proving lower closure theorems. We state here a few definitions, we restate for clarity a few theorems which had been proved in earlier papers, and we state a more general theorem which includes them, as well as some which have been encountered by Ioffe.

Let $\Lambda$ be a closed convex cone in $Z$ and let $\widetilde{Q}(y)$ denote $Q(y)+\Lambda$ for $y \in A$. We say that the sets $Q(y)$ are $\Lambda$-convex if $\widetilde{Q}(y) \equiv Q(y)$.

Note that for $Z=\mathbf{K}$ and $\Lambda=[r \mid r \geqslant 0], \Lambda$-convexity is the same as the "upper set property" used in [16], that is, $(\eta, z) \in Q(y)$ and $\bar{\eta} \geqslant \eta$ implies $(\bar{\eta}, z) \in Q(y)$.

Unless otherwise specified, whenever $Z$ is a finite dimensional space $\mathbf{R}^{\rho}$, we shall correspondingly take $\Lambda$ to be the positive octant

$$
\left[\eta\left|\eta=\left(\eta^{1}, \ldots, \eta^{\rho}\right)\right| \eta^{i} \geqslant 0, i=1, \ldots, \rho\right] .
$$

By a Nagumo function we mean a real-valued function $\phi(\xi)$ defined for $0 \leqslant \xi<+\infty$, which is bounded below, and satisfies $\phi(\xi) / \xi \rightarrow+\infty$ as $\xi \rightarrow+\infty$.

(3.i) Let $Z=\mathbf{R}$ and $B=\mathbf{R}^{n}$. For $y \in A \subset Y$ let $\widetilde{Q}(y)$ be a nonempty subset of $\mathbf{R}^{n+1}$ with the upper set property. For some $y_{0} \in A$ let $N_{\delta}\left(y_{0}\right)$ be a neighborhood of $y_{0}$ in $A$, and let us assume that there 
is a Nagumo function $\phi$ such that $(\eta, \zeta) \in \widetilde{Q}(y), \quad y \in N_{\delta}\left(y_{0}\right)$ implies $\eta \geqslant \phi(|\zeta|)$. If the sets $\widetilde{Q}(y)$ have property $(K)$ at $y_{0}$ and the set $\widetilde{Q}\left(y_{0}\right)$ is convex, then the sets $\widetilde{Q}(y)$ have property $(\mathrm{Q})$ at $y_{0}$.

(3.ii) Let $Z=\mathbf{R}$ and $B=\mathbf{R}^{n}$. For each $y \in A \subset Y$ let $Q_{0}(y)$ be a nonempty subset of $\mathbf{R}^{n}$. Let $T(y, \xi)$ be a real valued lower semicontinuous function on the set $S_{0}=\left[(y, \xi) \mid y \in A, \quad \xi \in Q_{0}(y)\right]$. For some $y_{0} \in A$ and neighborhood $N_{\delta}\left(y_{0}\right)$ of $y_{0}$ in $A$ let us assume that $T(y, \xi) \geqslant \phi(|\xi|)$ for all $y \in N_{\delta}\left(y_{0}\right), \quad \xi \in Q_{0}(y)$, and some Nagumo function $\phi$. If the sets $Q_{0}(y)$ have property (K)at $y_{0}$, and the set $\widetilde{Q}\left(y_{0}\right)$ is convex, then the sets $\widetilde{Q}(y)$ have property $(\mathrm{Q})$ at $y_{0}$.

Remark 1. The following example shows that the sets $\tilde{Q}(y)=\left[(\eta, \xi) \mid \eta \geqslant T(y . \xi), \quad \xi \in Q_{0}(y)\right]$ may not have property $(Q)$ without the growth condition expressed in Theorem (3.ii), and that this may occur even if $T$ is continuous, and convex in $\xi$ for every $y \in A$, and the sets $Q_{0}(y)$ have property (Q). Indeed, take

$$
A=[y \mid-1 \leqslant y \leqslant 1], \quad Q_{0}(y)=[\xi \mid-\infty<\xi<+\infty], \quad T(y, \xi)=y \xi .
$$

Then,

$$
\widetilde{Q}(0)=[(\eta, 0) \mid \eta \geqslant 0], \quad \widetilde{Q}(0, \delta)=\cup[\tilde{Q}(y),|y|<\delta]=[(\eta, \xi)|\eta \geqslant-| \delta \mid \xi, \quad \xi \in \mathbf{R}],
$$

and $\cap_{\delta} \operatorname{cl} \operatorname{co} \widetilde{Q}(0, \delta)=\mathbf{R}^{2} \neq \widetilde{Q}(0)(\mathrm{cf}[1$, p. 378]). However, it was shown [1, p. 379] that property (Q) of $\widetilde{Q}(y)$ at $y_{0}$ is guaranteed if, in addition, either the sets $Q_{0}(y), y \in A$, are all contained in a fixed ball in $\mathbf{R}^{n}$, or the function $T(y, \xi)$ in convex and seminormal in $\xi$ at every $y \in A$ and $\xi \in Q_{0}(y)$ (cf Section 5 in this paper). This property of seminormality is certainly satisficd if $T(y, \xi) / \xi \rightarrow+\infty$ as $|\xi| \rightarrow+\infty$ uniformly in $y \in N_{\delta}\left(y_{0}\right)$.

(3.iii) Let $Z=\mathbf{R}^{1+\rho}$ and $B=\mathbf{R}^{n}$. For $y \in A \subset Y$ let $\widetilde{Q}(y)$ be a nonempty subset of points $\left(\eta^{0}, \eta\right.$, $\xi)=\left(\eta^{0}, \eta^{1}, \ldots, \eta^{\rho}, \xi^{1}, \ldots, \xi^{n}\right) \in \mathbf{R}^{1+\rho+n}$ and let us assume $\widetilde{Q}(y)$ to be $\Lambda$-convex with respect to $\Lambda=\left[\left(\eta^{0}, \eta\right) \mid \eta^{0} \geqslant 0, \eta^{i} \geqslant 0, i=1, \ldots, \rho\right]$. For some $y_{0} \in A$ let $N_{\delta 0}\left(y_{0}\right)$ be a neighborhood of $y_{0}$ in $A$ and let us assume that $\left(\eta^{0}, \eta, \xi\right) \in \widetilde{Q}(y), \quad y \in N_{\delta 0}\left(y_{0}\right)$ implies $\eta^{0} \geqslant \phi(|\xi|), \eta^{i} \geqslant L, i=1, \ldots, \rho$, for some Nagumo function $\phi$ and real constant $L$. Then, if the sets $\widetilde{Q}(y)$ have property $(\mathrm{K})$ at $y_{0}$ and the set $\widetilde{Q}\left(y_{0}\right)$ is convex, then the sets $\widetilde{Q}(y)$ have property (Q) at $y_{0}$.

(3.iv) Let $Z=\mathbf{R}^{1+\rho}$ and $B=\mathbf{R}^{n}$. For each $y \in A\left\ulcorner Y\right.$ let $Q_{0}(y)$ be a nonempty subset of $\mathbf{R}^{n}$. Let $T_{i}(y, \xi), \quad i=0,1, \ldots, \rho$, be real-valued lower semicontinuous functions on the set $S_{0}=$ $\left[(y, \xi) \mid y \in A, \xi \in Q_{0}(y)\right]$, and for any $y \in A$ let $\widetilde{Q}(y)$ denote the set of points $\left(\eta^{0}, \eta, \xi\right)$ defined by $\left[\eta^{0} \geqslant\right.$ $\left.T_{0}(y, \xi), \eta^{i} \geqslant T_{i}(y, \xi), i=1, \ldots, \rho, \xi \in Q_{0}(y)\right]$. For some $y_{0} \in A$ and neighborhood $N_{\delta 0}\left(y_{0}\right)$ of $y_{0}$ in $A$ let us assume that $T_{0}(y, \xi) \geqslant \phi(|\xi|), T_{i}(y, \xi) \geqslant L$ for all $y \in N_{\delta 0}\left(y_{0}\right), \xi \in Q_{0}(y)$, and for some Nagumo function $\phi$ and real constant $L$. If the sets $Q(y)$ have property (K) at $y_{0}$ and the set $\widetilde{Q}\left(y_{0}\right)$ is convex, then the sets $\widetilde{Q}(y)$ have property $(\mathrm{Q})$ at $y_{0}$.

Theorems 3.i-iv can be proved by the same arguments used by Cesari in [2]. (For versions of the same theorems when $y$ is in a finite dimensional space see [17], (8.5). The proofs are essentially the same).

Part (b): Optimal Control formulation.

Here we present a theorem similar to those in part (a), but now in terms of control parameters. 
(3.v) Let $A \subset Y$ be nonempty. For each $y \in A$, let $U(y)$ be a given nonempty subset of a reflexive Banach space $(U,\|\|)$ equipped with the weak topology. Let $S=\{(y, u) \mid y \in A, u \in U(y)\}$ be given the product topology so that it has metric topology on $y$ and weak topology on $u$. Let $G(y, u)$, $g_{1}, \ldots, g_{\rho}, f_{1}, \ldots, f_{r}$ be given real valued functions defined on $A$. Let $G$ and $g_{1}, \ldots, g_{\rho}$ be nonnegative and lower semicontinuous on $S$ with respect to (metric $\times$ weak) topology. Let $f_{1}, \ldots, f_{r}$ be continuous on $S$. Let $f_{1}, \ldots, f_{r}$ and 1 be of slower growth than $G$ as $\|u\| \rightarrow \infty$, uniformly in $A$ (see remark below). Let the sets $U(y)$ satisfy property $(\mathrm{K})$ (with respect to weak topology on $U$ ) at $y=\bar{y}$ in $A$. If the set

$$
\widetilde{Q}_{G}(y)=\left\{(\tilde{\eta}, \xi) \mid \eta^{0} \geqslant G(y, u) ; \eta^{i} \geqslant g_{i}(y, u), i=1, \ldots, \rho, \xi^{i}=f_{i}(y, u), i=1, \ldots, r ; u \in U(y)\right\}
$$

is convex at $y=\bar{y}$, then the sets $\widetilde{Q}_{G}(y)$ satisfy property $(\mathrm{Q})$ at $y=\bar{y}$.

Remark 2. A function $f$ is said to be of slower growth than $G$ as $\|u\| \rightarrow \infty$ uniformly in $A$ provided (a) given $\varepsilon>0$ there is an $N=N(\varepsilon)>0$ such that $\|u\| \geqslant N, u \in U(y)$ implies $|f(y, u)| \leqslant \varepsilon G(y, u)$, for all $y \in A$ and (b) $f(y, u)$ remains bounded for $y \in A$ and $\|u\|$ bounded. Due to the local character of the conclusion, it would be enough to require the slow growth to be uniform only in a neighborhood of $\bar{y}$ in $A$.

\section{Remark 3.}

(i) Theorem (3.v) was essentially proved directly in [2].

(ii) Theorem (3.i) implies Theorem (3.v). First let us prove that the sets $\widetilde{Q}_{G}(y)$ have property (K) at $\bar{y}$, that is, that $\wedge_{\delta} \mathrm{cl} \widetilde{Q}_{G}(\bar{y}, \delta)=\widetilde{Q}_{G}(\bar{y})$. For $\tilde{\eta}=\left(\eta^{0}, \eta\right)=\left(\eta^{0}, \eta^{1}, \ldots, \eta^{\prime}\right)$, it is enough to prove that, if $\left(\bar{\eta}^{0}, \bar{\eta}, \bar{\xi}\right) \in \cap_{\bar{\delta}} \operatorname{cl} \widetilde{Q}_{G}(\bar{\eta}, \delta)$, then $\left.\bar{\eta}^{0}, \bar{\eta}, \bar{\xi}\right) \in \widetilde{Q}_{G}(\bar{y})$. Indeed, $\left(\bar{\eta}^{0}, \bar{\eta}, \bar{\xi}\right) \in \operatorname{cl} \widetilde{Q}_{G}(\bar{y}, \delta)$ for all $\delta>0$, and there is a sequence $\left(\eta_{k}^{0}, \eta_{k}, \xi_{k}\right) \in Q_{r_{r}}\left(y_{k}\right)$ with $y_{k} \in A, \quad y_{k} \rightarrow \bar{y}, \quad \eta_{k}^{0} \rightarrow \bar{\eta}^{0}, \eta_{k} \rightarrow \bar{\eta}, \xi_{k} \rightarrow \bar{\xi}$ as $k \rightarrow \infty$. Thus, there are also points $u_{k}$ such that

$$
u_{k} \in U\left(y_{k}\right), \eta_{k}^{0} \geqslant G\left(y_{k}, u_{k}\right), \eta_{k}^{i} \geqslant g_{i}\left(y_{k}, u_{k}\right), \xi_{k}^{i}=f_{i}\left(y_{k}, u_{k}\right) \text {. }
$$

Here $\eta_{k}^{0} \rightarrow \bar{\eta}^{0}$ hence $\left[\eta_{k}^{0}\right]$ is a bounded sequence, and by the growth property on $G$ we derive that $\left[u_{k}\right]$ is also a bounded sequence of elements $\left[u_{k}\right]$ of the reflexive Banach space $(u,\|\|$,$) . Hence,$ there is a subsequence, say still $[k]$, such that $u_{k} \rightarrow \bar{u} \in U$ weakly in the Banach space $U$. In other words, $\vec{u} \in \cap_{\delta} \mathrm{cl}_{w} U(\bar{y}, \delta)$ where $\mathrm{cl}_{w}$ means weak closure. Since by hypothesis, the sets $U(y)$ have property $(\mathrm{K})$ with respect to the weak topology in $U$, we conclude that $\bar{u} \in U(\bar{y})$. Hence, $(\bar{y}, \bar{u}) \in S$, and then $\eta^{0} \geqslant G(\bar{y}, \bar{u}), \eta^{i} \geqslant g_{i}(\bar{y}, \bar{u}), \xi^{i}=f_{i}(\bar{y}, \bar{u})$, or $\left(\eta^{0}, \bar{\eta}, \xi\right) \in \widetilde{Q}_{G}(y)$. We have proved that the sets $\widetilde{Q}_{G}(y)$ have property $(\mathrm{K})$ at $\bar{y}$. Let us prove that there is a function $\phi(\xi)$ with $\phi(\xi) / \xi \rightarrow+\infty$ as $\xi \rightarrow+\infty$, with $G(y, u) \geqslant \phi(|f(y, u)|)$ for all $y \in A, \quad u \in U(y)$. Indeed in the opposite case, there would be a constant $C>0$ and a sequence $\left(y_{k}, u_{k}\right)$ with

$$
y_{k} \in A, \quad u_{k} \in U\left(y_{k}\right), \quad \xi_{k}=f\left(y_{k}, u_{k}\right), \quad \eta_{k}^{0}=G\left(y_{k}, u_{k}\right), \quad \eta_{k}^{0} \leqslant C \xi_{k}, \quad \xi_{k} \rightarrow+\infty \quad \text { as } \quad k \rightarrow \infty .
$$

Thus, $\left\|u_{k}\right\| \rightarrow+\infty$, while $G\left(y_{k}, u_{k}\right) \leqslant C f\left(y_{k}, u_{k}\right)$, a contradiction. Now Theorem (3.i) applies.

(iii) Theorem (3.v) implies Theorem (3.i). Indeed, if we take

then we have

$$
f_{i}(y, u)=u^{i}=\xi^{i}, \quad i=1, \ldots, r, \quad U=\mathbf{R}^{r},
$$

$$
\begin{aligned}
Q_{G}(y, u) & =\left[\eta^{0} \geqslant G(y, u), \eta^{i} \geqslant g_{i}(y, u), \xi^{i}=u^{i}\right] \\
& =\left[\eta^{0} \geqslant G(y, \xi), \eta^{i} \geqslant g_{i}(y, \xi), \xi \in U(y)\right]
\end{aligned}
$$


and we have Theorem (3.i) but for the notations. We see that Theorem (3.i) and Theorem (3.v), the latter essentially proved in [2], are equivalent.

Remark 4. If the Banach space $U$ in Theorem (3.v) is finite dimensional then weak and strong topologies coincide so that Theorem (3.v) reduces to Theorem (2.2.ii) of [2] in the particular case $Y=\mathbf{R}^{1+n}$. The proof on pp. 521-524 of [2] needs no major changes, even in the general situation stated above.

In [4] Ioffe used the basic argument found in the proof of [2] cited above, in order to prove a lower semicontinuity theorem. In [8] the present authors also used the same argument to prove the more general lower closure theorems (3.v) by explicit requirement of mere property (K). Property (Q) for suitable auxiliary sets then follows as shown by the theorems above (3.iv), and many variants (cf [8], Remark (3.6), p. 229, and [17]).

\section{DUALITY AND A CHARACTERIZATION OF PROPERTY (Q)}

We report here, for clarity, the main points of the duality operation in convex analysis, and a characterization of property $(Q)$ in terms of duality operations recently proved by Goodman [3] and reported in [17].

Let $B$ be a Banach space over the reals, let $B^{*}$ be the dual of $B$, and let $\langle\xi, \xi\rangle$ denote the linear operation $\zeta \in B^{*}$ applied to the element $\zeta \in B$.

In this and the next section we shall consider extended real valued functions $T$ on $B$, that is, those for which the values $+\infty$ and $-\infty$ are allowed. Let $Q$ denote the subset of $B$ where $T \xi \neq+\infty$. We denote by epigraph of $T$, or $\widetilde{Q}=$ epi $T$ the subset of $R \times B$ defined by $\widetilde{Q}=$ epi $T=[(\eta, \xi) \mid$ $\eta \geqslant T(\xi), \xi \in B, \eta \in \mathbf{R}]$. Then we have also $T \xi=\operatorname{Inf}[\eta \mid(\eta, \xi) \in \widetilde{Q}], \xi \in B$, with the understanding that $[\eta \geqslant T(\xi), \xi \in B, \eta \in \mathbf{R}]=\phi$ is the empty set if $\xi \in B-Q$, and that $[\eta \geqslant T(\xi), \xi \in B, \eta \in \mathbf{R}]=$ $\mathbf{R}$ if $\xi \in Q, \quad T(\xi)=-\infty$. The concept of convexity of such a function $T$ on $B$ applies as usual.

(4.i) The set $\widetilde{Q}=$ epi $T$ is convex if and only if $T$ is convex; the set $\widetilde{Q}=$ epi $T$ is closed if and only if $T$ is lower semicontinuous on $B$.

We denote now by $\{h(\xi)\}$ the class of all affine functions on $B$, that is, $h(\xi)=\rho+\langle\zeta, \xi\rangle$, where $\rho \in \mathbf{R}$ and $\zeta \in B^{*}$. We say that $h \in\{h(\xi)\}$ is a supporting plane for $T$ if $h(\xi) \leqslant T \xi$ for all $\xi \in B$. By the symbol $h \leqslant T$ we mean that $h(\xi) \leqslant T \xi$ for all $\xi \in B$. It is easy to prove that

(4.ii) If $T$ is convex, and lower semicontinuous in $B$ with $T \xi \not \equiv+\propto, T \xi \neq-\infty$, for all $\xi$, then $T \xi=\operatorname{Sup}[h(\xi) \mid h \leqslant T]$.

In other words, $T$ can be recovered from its supporting planes. In other words, if $\left\{S^{+}\right\}$is the collection of all half spaces containing $\widetilde{Q}=$ epi $T$, or $S^{+}=[(\eta, \xi) \mid \eta \geqslant h(\xi), \xi \in B]$ for all $h \in\{h\}$ with $h \leqslant T$, then $\widetilde{Q}=\cap S^{+}$.

For extended real functions $T \xi, \xi \in B$, we shall need below the closure operation, or cl $T$, which is defined by means of the relation epi(cl $T)=\operatorname{cl}($ epi $T)$.

Given an extended real function $T \xi, \xi \in B$, we now define as the dual $T^{*}$ of $T$ the extended real function $T^{*} \zeta, \zeta \in B^{*}$, by taking

$$
T^{* \zeta}=\operatorname{Sup}[\langle\zeta, \xi\rangle-T \xi \mid \xi \in B], \zeta \in B^{*} .
$$

It can be easily proved that 
(4.iii) If $T \xi, \xi \in B$, is an extended real function, convex and lower semicontinuous in $B$, with $T \xi \not \equiv+\infty, T \xi \neq-\infty$ for all $\xi$, then $T^{*} \zeta, \zeta \in B^{*}$ is also an extended real function, convex and lower semicontinuous in $B^{*}$ with $T^{*} \zeta \not \equiv+\infty, T^{*} \zeta \neq-\infty$ for all $\zeta$, and

$$
\widetilde{Q}=\operatorname{epi} T=\cap_{\zeta \in B^{*}}\left[(\eta, \xi) \mid \eta \geqslant-T^{* \zeta}+\langle\zeta, \xi\rangle, \xi \in B\right] .
$$

Moreover, if $T$ is as in (4.iii), then we can define $\left(T^{*}\right)^{*}$ or $T^{* *}$ and it can be easily proved that

(4.iv) If $T$ is an extended function, convex and lower semicontinuous on $B$, with $T \xi \not \equiv+\infty$, $T \xi \neq-\infty$ for all $\xi$, then $T^{* *}=T$.

If $\Gamma=\Gamma(B)$ denotes the class of all extended real function $T \xi, \xi \in B$, convex and lower semicontinuous in $B$, then we see from the above that the operation $T \rightarrow T^{*}$ is one-one, and maps $\Gamma(B)$ into $\Gamma\left(B^{*}\right)$. If $\left[T_{i} \xi, \xi \in B\right], \quad i \in I$, is a family of functions $T_{i} \in \Gamma(B)$ depending on an index $i$ ranging over an index set $I$, then we denote by $\Lambda$ and $V$ the lattice operations

$$
\begin{aligned}
& \left(V_{i} T_{i}\right) \xi=\operatorname{Sup}_{i} T_{i} \xi \\
& \left(\Lambda_{i} T_{i}\right) \xi=\operatorname{Sup}\left[h(\xi) \mid h \leqslant T_{i} \text { for all } i \in I\right] .
\end{aligned}
$$

For sets, that is, for the corresponding epigraphs $Q_{i}=$ epi $T_{i}, \quad i \in I$, we may use analogous symbols

$$
\begin{aligned}
& \stackrel{\circ}{V_{i}} Q_{i}=\operatorname{clco} \cup_{i} Q_{i}, \\
& \stackrel{\circ}{\Lambda}_{i} Q_{i}=\cap_{i} Q_{i} .
\end{aligned}
$$

With these notations, and functions $T_{i} \in \Gamma$ we have

$$
\stackrel{\circ}{\Lambda}_{i}\left(\text { epi } T_{i}\right)=\operatorname{epi} V_{i} T_{i}, \quad V_{i}\left(\text { epi } T_{i}\right)=\operatorname{epi} \Lambda_{i} T_{i}
$$

and furthermore it is easy to prove that

(4.v) If $T_{i} \in \Gamma$ for all $i \in I$ and $\left(V_{i} T_{i}\right) \xi \not \equiv+\infty$, then $V_{i} T_{i} \in \Gamma$. If $\left(\Lambda_{i} T_{i}\right) \xi \neq-\infty$ for all $\xi$, then $\Lambda_{i} T_{i} \in \Gamma$.

(4.vi) If $T_{i} \in \Gamma$ for all $i \in I$, then

$$
\left(\Lambda_{i} T_{i}\right)^{*}=V_{i} T_{i}^{*}, \quad\left(V_{i} T_{i}\right)^{*}=\Lambda_{i} T_{i}^{*}
$$

We consider now with Goodman the case where $I$ is an ordered set, that is, $I$ is ordered by an order relation, with a "least" element $\omega$, such that (1) $i<j, j<k$ implies $i<k$; (2) given $i, j \in I$, $i, j \neq \omega$, there is $k \in I, k \neq \omega$, such that $k<i, k<j$. Then, instead of the operation "Sup" we may consider the operation corresponding to "lim sup" in the present terms. Thus, instead of taking, say, $\stackrel{\circ}{V}_{i} Q_{i}=\mathrm{cl} \operatorname{co} \cup_{i} Q_{i}$, we may take

$$
\check{\Lambda}_{\lambda \neq \omega} \stackrel{\circ}{V}_{i<\lambda} Q_{i}=\bigcap_{\lambda \neq \omega} \operatorname{clco} \bigcup_{i<\lambda} Q_{i}
$$

and instead of, say $\Lambda_{i} T_{i}$, we may take

$$
V_{\lambda \neq \omega} \Lambda_{i<\lambda} T_{i}(\xi)=\operatorname{Sup}_{\lambda \neq \omega}\left[h(\xi) \mid h<T_{i}, i<\lambda\right] .
$$

In these conditions, we note that

$$
\widetilde{T}_{\lambda} \xi=\left(V_{i<\lambda} T_{i}\right) \xi=\operatorname{Sup}\left[T_{i} \xi, i<\lambda, \xi \in B\right],
$$


is a convex lower semicontinuous function of $\xi$ which depends monotonically on $\lambda$. The following lemma, where use is made of the closure operation defined above, is relevant.

(4.vii) (Goodman [3]). If the index set $I$ is ordered then

$$
\operatorname{clinf} \tilde{T}_{\lambda} \xi=\Lambda_{\lambda} \tilde{T}_{\lambda} \xi
$$

We consider now the case where we have a family of sets

$$
\widetilde{Q}(x)=\text { epi } T(x, \xi)=[(\eta, \xi) \mid \eta \geqslant T(x, \xi), \xi \in B\rfloor
$$

indexed by $x \in A$, a subset of a metric space $X$ with distance function $d\left(x, x_{0}\right)$ from a fixed point $x_{0} \in A$. That is, we say that $x<y$ if $x, y \in A$ and $d\left(x, x_{0}\right)<d\left(y, x_{0}\right)$. With this understanding the "lim sup" operation mentioned above becomes

$$
\stackrel{\circ}{\delta>0} \stackrel{\circ}{V}\left[\widetilde{Q}(x), d\left(x, x_{0}\right)<\delta\right]=\bigcap_{\delta>0} \operatorname{cl} \operatorname{co} \bigcup\left[\tilde{Q}(x), d\left(x, x_{0}\right)<\delta\right] .
$$

Thus, property $(\mathrm{Q})$ at $x_{0}$ reduces to the requirement that

$$
\widetilde{Q}\left(x_{0}\right)=\stackrel{\circ}{\Lambda}>0_{\mathrm{V}} \mathrm{\vee}\left[\widetilde{Q}(x), d\left(x, x_{0}\right)<\delta\right]
$$

or equivalently

$$
\text { epi } T\left(x_{0}, \xi\right)=\stackrel{\circ}{\Lambda}_{\delta>0} \stackrel{\circ}{\mathrm{V}}\left[\text { epi } T(x, \xi), d\left(x, x_{0}\right)<\delta\right] \text {, }
$$

or

$$
T\left(x_{0} \xi\right)=V_{\delta>0} \Lambda\left[T(x, \xi), d\left(x, x_{0}\right)<\delta\right]
$$

We have the final result

(4.viii) THEOREM (Goodman [3]). If $T(x, \xi)>-\infty$ for all $(x, \xi) \in A \times B$, then the sets $\widetilde{Q}(x)=$ epi $T(x, \xi)$ have property (Q) at $x_{0}$ if and only if

$$
T^{*}\left(x_{0}, \xi\right)=\Lambda_{\delta} V\left[T^{*}(x), d\left(x, x_{0}\right)<\delta\right],
$$

or equivalently

or equivalently

$$
T^{*}\left(x_{0}, \xi\right)=\operatorname{cl}\left[\limsup _{x \rightarrow x_{0}} T^{*}(x, \xi)\right]
$$

$$
T\left(x_{0}, \xi\right)=\left[\limsup _{x \rightarrow x_{0}} T^{*}\left(x_{0}, \xi\right)\right]^{*}
$$

Detailed proofs of the statements in this section have been given in a slightly less general context in $[17,(8.11)]$. The proofs hold in the present context without changes.

Remark. In this section we have considered extended real valued functions and their epigraphs. Dually, one could start with subsets $\widetilde{Q} \subset R \times B$ with the property that $(\eta, \xi) \in \widetilde{Q}, \eta^{\prime} \geqslant \eta$, implies that $\left(\eta^{\prime}, \xi\right) \in \widetilde{Q}$, and correspondingly define $T: B \rightarrow R \cup\{ \pm \infty\}$ as $T \xi=\inf \{\eta \mid(\eta, \xi) \in \widetilde{Q}\}$ with the understanding that $T \xi=+\infty$ if there is no $\eta$ with $(\eta, \xi) \in \widetilde{Q}$ and $T \xi=-\infty$ if $(\eta, \tilde{\xi}) \in \tilde{Q}$ for all $\eta \in \mathbf{R}$. If $\widetilde{Q}$ is closed, then $\widetilde{Q}=$ epi $T$. This process will be pursued further in the next section. 
We shall now make two observations of interest in this connection. First, if $\widetilde{Q}^{*}$ denotes epi $T^{*}$ then clearly,

$$
\widetilde{Q}^{*}=\cap\{(\theta, \check{\zeta}) \mid\langle\zeta, \xi\rangle \leqslant T \xi+\theta, \xi \in B\} .
$$

Also, if $T \xi=-\infty$ then $\widetilde{Q}^{*}$ is empty. Finally, $\widetilde{Q}=\widetilde{Q}^{* *}=\cap\left\{(\eta, \xi) \mid\langle\zeta, \xi\rangle \leqslant \eta+T^{*}, \zeta \in B^{*}\right\}$; (Goodman [3], Suryanarayana [22], Denkowski [23]).

For the second observation, let us assume that the sets $\widetilde{Q}$ depend on $t \in G$, so that $T(t, \xi)=$ $\inf \{\eta \mid(\eta, \xi) \in \widetilde{Q}(t)\}$ and $\widetilde{Q}^{* *}=\widetilde{Q}$ reads as follows:

$$
\widetilde{Q}(t)=\cap\left\{(\eta, \xi) \mid-\eta+\langle\zeta, \xi\rangle \leqslant \theta ; \quad(\theta, \zeta) \in \operatorname{gr}\left(T^{*}(t, \cdot)\right)\right\},
$$

where $\operatorname{gr}\left(T^{*}(t, \cdot)\right)=\left\{\left(T^{*}(t, \zeta), \zeta\right): \zeta \in B^{*}\right\}$ is the graph of $T^{*}(t, \zeta)$ as a function of $\zeta$ alone. It is easy to see that we get the same result if we take the intersection of the above sets with $(\theta, \zeta)$ now ranging over the epigraph of $T^{*}(t, \cdot)$, namely, epi $T^{*}(t)=\left\{(\theta, \zeta) \mid \theta \geqslant T^{*}(t, \zeta), \zeta \in B^{*}\right\}$. Using Castaing's representation [18], for measurable multivalued mappings defined over a separable Banach space $B$, one obtains: Let $B$ be separable and let there exist $\zeta_{0} \in L_{\infty}(G)$ and $\theta_{0} \in L_{1}(G)$ such that $T^{*}(t$, $\left.\zeta_{0}(t)\right) \leqslant \theta_{0}(t)$. Then

$$
\widetilde{Q}(t)=\bigcap_{i=1}^{\infty}\left\{(\eta, \xi) \mid-\eta+\left\langle\zeta_{i}(t), \xi\right\rangle \leqslant \theta_{i}(t)\right\}
$$

for some sequence $\left(\zeta_{i}, \theta_{i}\right) \in L_{\infty}\left(G, B^{*}\right) \times L_{1}(G, B)$ with $\theta_{i}(t) \geqslant T^{*}\left(t, \zeta_{i}(t)\right)$.

\section{SEMINORMALITY AND ANOTHER CHARACTERIZATION OF PROPERTY (Q)}

We report here the concept of seminormality $[1,10-12]$ for convex functions, and a corresponding characterization of property $(Q)$ recently proved by Cesari [10]. A stronger form of seminormality had been used by Tonelli and later by McShane. Again, let $B$ be a Banach space over the reals $\mathbf{R}$, let $B^{*}$ be the dual of $B$ and let $\langle\zeta, \xi\rangle$ denote the linear operation $\zeta \in B^{*}$ applied to the elements $\xi \in B$. For every $y \in A \subset Y$ let $\widetilde{Q}(y)$ denote a given subset of $\mathbf{R} \times B$ and let $Q(y)$ denote the projection of $\widetilde{Q}(y)$ on $B$. Moreover we assume that $\widetilde{Q}(y)=$ epi $T(y,$.$) where T$ is as in the Remark of Section 4; in other words, for every $y \in A, T(y, \xi)$, as a function of $\xi$ in $B$, is an extended real function on $B$, and we explicitly assume that $T(y, \xi) \neq-\infty$ for all $y$ and $\xi$, that for every $y \in A$ the sets $\widetilde{Q}(y)$ and $Q(y)$ are not empty, hence $T(y, \xi) \neq \equiv+\infty$ for any $y \in A$, and $T(y, \xi)=+\infty$ for all $\xi \in B-Q(y), y \in A$. Property (Q) at any point $y_{0} \in A$ for the sets $\widetilde{Q}(y)$ is defined by requiring

$$
\widetilde{Q}\left(y_{0}\right)=\bigcap_{\delta>0} \operatorname{clco} \bigcup\left[\widetilde{Q}(y), d\left(y, y_{0}\right)<\delta\right]=\bigcap_{\delta} \operatorname{cl} \operatorname{co} \tilde{Q}\left(y_{0}, \delta\right) .
$$

Thus, if $\left(\eta_{0}, \xi_{0}\right) \in \bigcap_{\delta>0} \operatorname{cl} \operatorname{co} \widetilde{Q}\left(y_{0}, \delta\right)$, then necessarily $\left(\eta_{0}, \xi_{0}\right) \in \widetilde{Q}\left(y_{0}\right)$ and hence $\xi_{0} \in Q\left(y_{0}\right)$. For a given $y_{0} \in A$ we denote by $(\alpha)$ the requirement

$$
(\alpha) \text { If }\left(\eta_{0}, \xi_{0}\right) \in \bigcap_{\delta>0} \operatorname{cl} \operatorname{co} \widetilde{Q}\left(y_{0}, \delta\right) \text { then } \xi_{0} \in Q\left(y_{0}\right) \text {. }
$$

Thus, $(\alpha)$ is a necessary condition for property $(\mathrm{Q})$ at $y_{0}$. On the other hand, $(\alpha)$ is identically satisfied whenever $Q(y)=B$ for all $y \in A$. This situation occurs in the classical problems of the calculus of variations with $Q(y)=B=\mathbf{R}^{n}$. We shall now introduce the following condition (X) at $y_{0} \in A$ : 
(X) Given $\varepsilon>0$ and any $\xi_{0} \in Q\left(y_{0}\right)$, there is a $\delta>0$ and an affine map $h(\xi), \xi \in B$, such that

$$
\begin{aligned}
& \left(\mathrm{X}_{1}\right) T(y, \xi) \geqslant h(\xi) \text { for all } \xi \in B \text { and all } y \in A \text { with } d\left(y, y_{0}\right)<\delta ; \\
& \left(\mathrm{X}_{2}\right) T\left(y_{0}, \xi_{0}\right)<h\left(\xi_{0}\right)+\varepsilon .
\end{aligned}
$$

We shall say that $T(y, \xi)$ is seminormal at the point $y_{0} \in A$ if conditions $(\alpha)$ and $(\mathrm{X})$ hold.

A slightly stronger condition is as follows:

$\left(\mathrm{X}^{\prime}\right)$ given $\varepsilon>0$ and any $\xi_{0} \in Q\left(y_{0}\right)$, there are numbers $\delta>0, \quad v>0$, and an affine map $h(\xi), \quad \xi \in B$, such that

$\left(X_{1}^{\prime}\right) T(y, \xi) \geqslant h(\xi)+v\left|y-y_{0}\right|$ for all $\xi \in B$ and all $y \in A$ with $d\left(y-y_{0}\right)<\delta$;

$\left(\mathrm{X}_{2}^{\prime}\right)$ the same as $\left(\mathrm{X}_{2}\right)$.

We shall say that $T(y, \xi)$ is normal at the point $y_{0} \in A$ if conditions $(\alpha)$ and $\left(\mathrm{X}^{\prime}\right)$ hold.

(5.i) If conditions $(\alpha)$ and $(\mathrm{X})$ hold at the point $y_{0} \in A$, then the set $\widetilde{Q}\left(y_{0}\right)$ is closed and convex, and the sets $\widetilde{Q}(y)$ have property $(\mathrm{Q})$ at $y_{0}$.

Proof. If $\left(\eta_{0}, \xi_{0}\right) \in \cap_{\delta>0}$ cl co $\widetilde{Q}\left(y_{0}, \delta\right)$, then, by $(\alpha), \xi_{0} \in Q\left(y_{0}\right)$, and corresponding to this $\xi_{0}$ and arbitrary $\varepsilon>0$ then, by $\left(\mathrm{X}_{1}\right)$, there are a number $\delta>0$ and an affine function $h(\xi), \quad \xi \in B$, such that

$$
\widetilde{Q}\left(y_{0}, \delta\right)=\cup\left[\widetilde{Q}(y), d\left(y, y_{0}\right)<\delta\right] \subset[(\eta, \xi) \mid \eta \geqslant h(\xi), \xi \in B] .
$$

Since the right hand side is necessarily closed and convex in $R \times B$ we obtain

$$
\left(\eta_{0}, \xi_{0}\right) \in \cap_{\delta>0} \operatorname{clco} \widetilde{Q}\left(y_{0}, \delta\right) \subset[(\eta, \xi) \mid \eta \geqslant h(\xi), \xi \in B],
$$

that is, $\eta_{0} \geqslant h\left(\xi_{0}\right)$. Using $\left(\mathrm{X}_{2}\right)$ we have $\eta_{0} \geqslant T\left(y_{0}, \xi_{0}\right)-\varepsilon$, and since $\varepsilon$ is arbitrary we also have $\eta_{0} \geqslant T\left(y_{0}, \xi_{0}\right)$, and then $\left(\eta_{0}, \xi_{0}\right) \in \widetilde{Q}\left(y_{0}\right)$. This proves (5.i).

Remark 1. The above theorem can be generalized to the case where $\eta \in \mathbf{R}^{\rho}$ instead ot $\mathbf{R}^{1}$.

Remark 2. Theorem (5.i) also holds with the following modifications. First let us consider sets of the form

$$
\widetilde{Q}(y)=\{(\eta, \xi)|| \eta \geqslant T(y, u), \xi=f(y, u), u \in U(y)\}
$$

and let us assume that

$\left(\alpha^{\prime \prime}\right)$ If $\left(\eta_{0}, \xi_{0}\right) \in \cap_{\delta>0} \mathrm{cl}$ co $\widetilde{Q}\left(y_{0}, \delta\right)$ then $\xi_{0}=f\left(y_{0}, u_{0}\right)$ for some $u_{0} \in U\left(y_{0}\right)\left(\mathrm{X}^{\prime \prime}\right)$. Given $\varepsilon>0$ and $u_{0}$ as in $\left(\alpha^{\prime \prime}\right)$, there are numbers $\delta>0, r$ and a vector $\zeta \in B^{*}$ such that

$$
\begin{aligned}
& \left(\mathrm{X}_{1}^{\prime \prime}\right) T(y, u) \geqslant r+\langle\zeta, f(y, u)\rangle \text { for all } u \in U(y) \text { and all } y \text { with } d\left(y, y_{0}\right) \leqslant \delta ; \\
& \left(\mathrm{X}_{2}^{\prime \prime}\right) T\left(y_{0}, u_{0}\right) \leqslant r+\left\langle\zeta, f\left(y_{0}, u_{0}\right)\right\rangle+\varepsilon .
\end{aligned}
$$

Now theorem (5.i) holds in modified form as follows (cf [10]):

(5.i) If conditions $\left(\alpha^{\prime \prime}\right)$ and $\left(\mathrm{X}^{\prime \prime}\right)$ hold at the point $y_{0} \in A$, then the set $\widetilde{Q}\left(y_{0}\right)$ is closed and convex and the sets $\widetilde{Q}(y)$ satisfy property $(Q)$ at $y_{0}$.

Remark 3. Theorem (5.i) is a generalization of theorem 4.i of [10] where the spaces were taken to be Euclidean and Caratheodory convexity theorem was used. While the above theorem shows that we do not need finite dimensionality in $\xi$, we do use finite dimensionality to prove the 
converse statement (namely, by requiring the compactness of the unit ball) and this is theorem (9.i) of $[10]$ :

(5.ii) (Cesari [10] (9.i)) If for a given $y_{0} \in A$ we have $T\left(y_{0}, \xi\right)>-\infty$ for all $\xi \in Q\left(y_{0}\right) \subset \mathbf{R}^{n}$, then the sets $\widetilde{Q}(y)$ have property $(\mathrm{Q})$ at $y_{0}$ if and only if properties $(\alpha)$ and $(\mathrm{X})$ hold at $y_{0}$.

Thus, for $B=\mathbf{R}^{n}$, property (Q) is equivalent to the seminormality conditions $(\alpha)$ and $(\mathrm{X})$. For extensions of this theorem to locally convex spaces cf Denkowski [23]. For further work on property $(Q)$ see Cesari $[12,15]$, Cesari and Suryanarayana $[8,9,14]$, Kaiser and Suryanarayana [16], Goodman [3], Olech [6], and for connections with lattice theory in topology see Suryanarayana $[22]$.

\section{A CRITERION FOR PROPERTY (Q) IN TERMS OF ASYMPTOTIC CONES}

In Sections 3, 4, 5 we have already stated various criteria for property (Q) in terms of growth or seminormality conditions. Others will be stated in Section 7 in terms of analytic conditions. Here is a criterion due to Olech [5] in terms of asymptotic cones, related to earlier work of Cesari [10] (cf also [17]).

If $T(y, \xi)$ is a continuous function on $A \times \mathbf{R}^{n}, A$ an open subset of $\mathbf{R}^{r}$, and $T(y, \xi)$ is a convex function of $\xi$ in $\mathbf{R}^{n}$ for all $y \in A$, then it is known that $T(y, \xi)$ is normal at $y_{0}$, that is, both conditions $(\alpha)$ and $\left(\mathrm{X}^{\prime}\right)$ hold at $y_{0}$, if and only if the graph of $T\left(y_{0}, \xi\right)$, or $\left[(\eta, \xi) \mid \eta \geqslant T\left(y_{0}, \xi\right), \xi \in \mathbf{R}^{n}, \eta \in \mathbf{R}\right]$ contains no straight line. This was proved by Tonelli under smoothness hypotheses, and recently proved by Turner under the hypotheses of continuity (see, e.g., a proof in [10] (5.ix)).

In general, for functions $T$ which may take the value $+\infty$, that is, for sets $\widetilde{Q}(y)$ in $\mathbf{R} \times \mathbf{R}^{n}$ whose projection $Q(y)$ on $\mathbf{R}^{n}$ may not be all of $\mathbf{R}^{n}$, the statement above needs further conditions. Again we assume $T(y, \xi)$ continuous on $M_{0}=[(y, \xi) \mid y \in A, \xi \in Q(y)]$ and that, for every $y \in A$, $T(y, \xi)$ is convex in $\xi$ in the set $Q(y)$.

Let us consider again subsets $\widetilde{Q}(y), y \in A$, of $\mathbf{R}^{n+1}$. For any $y \in A$, let $C(y)$ denote the set

$$
C(y)=\left[\tilde{c} \in \mathbf{R}^{n+1} \mid \tilde{z}+\lambda \tilde{c} \in \tilde{Q}(y) \text { for all } \lambda \geqslant 0 \text { and } \tilde{z} \in \tilde{Q}(y)\right] .
$$

Obviously, $C(y)$ is a cone of vertex $\tau=0$ in $\mathbf{R}^{n+1}$. The set $C(y)$ is said to be the asymptotic cone of the set $\widetilde{Q}(y)$. It is easy to see that, if $\widetilde{Q}(y)$ is convex, so is $C(y)$; if $\widetilde{Q}(y)$ is closed, so is $C(y)$. Let $\Gamma$ denote the particular cone in $\mathbf{R}^{n+1}$ made up of the only positive half axis $z^{0} \geqslant 0$, or $\Gamma=[\tilde{z}=$ $\left.\left(z^{0}, z\right) \mid z^{0} \geqslant 0, z=0\right]$. Then $\Gamma \subset C(y)$ for all $y \in A$. We shall denote by $C^{0}(y)$, or polar cone of $C(y)$ the set $C^{0}(y)=\left[\tilde{d} \in \mathbf{R}^{n+1} \mid \tilde{d} . \tilde{c} \leqslant 0\right.$ for all $\left.\tilde{c} \in C(y)\right]$. If $C(y)=\Gamma$, then obviously

$$
C^{0}=\Gamma^{0}=\left[d=\left(d^{0}, d\right) \in \mathbf{R}^{n+1} \mid d^{0} \leqslant 0, d \in \mathbf{R}^{n}\right] .
$$

(6.i) (Olech [5]) If the sets $\widetilde{Q}(y)$ satisfy property (K) in $A$ (thus, they are closed and their graph is closed), if they are convex and do not contain any straight line, if the asymptotic cone is constant, or $C(y)=C=$ const, and for each number $r>0$ and point $\tilde{d} \in$ int $C^{0}, C^{0}$ the polar cone, we have

$$
\operatorname{Sup}_{y \in A} \operatorname{Sup}_{\tilde{z} \in Q(y)}(\tilde{d} \cdot \tilde{z})<+\infty,
$$

then the sets $\widetilde{Q}(y)$ satisfy property $(\mathrm{Q})$ in $A$. 


\section{THE CONDITION (D) IN BANACH SPACES}

(a) A necessary and sufficient condition for property (D).

For the sake of simplicity we use the same notations as in Section 2(b), and particularly statement (2.viii). Thus, $U(t)$ depends only on $t$,

$$
u_{k}(t) \in U(t) \subset U, \quad y_{k}(t) \in A(t) \subset Y, \quad \tilde{f}=\left(f_{0}, f\right),
$$

$y_{k}(t) \rightarrow y(t)$ in measure in $G$ as $k \rightarrow \infty$, and $\delta_{k}(t)=\left(\delta_{k}^{0}, \delta_{k}\right)$ is defined by

$$
\tilde{\delta}_{k}(t)=f\left(t, y_{k}(t), u_{k}(t)\right)-\tilde{f}\left(t, y(t), u_{k}(t)\right)
$$

(7.i) $\tilde{\delta}_{k}(t) \rightarrow 0$ strongly in $L_{1}(G, Y \times B)$ if and only if the same functions $\tilde{\delta}_{k}(t)$ are equiabsolutely integrable in $G$.

This statement was proved in [14] (3.1) in a slightly more elementary situation, but the proof is the same.

(7.ii) $\tilde{\delta}_{k}(t) \rightarrow 0$ implies the following weak form of property (Q) namely, there is a subsequence $\left[k_{s}\right]$ such that

$$
\widetilde{Q}(t, y(t)) \supset \cap_{h=1}^{\infty} \operatorname{cl} \operatorname{co}\left[\bigcup_{s=h}^{\infty} \tilde{f}\left(t, y_{k_{s}}(t), u_{k_{s}}(t)\right], t \in G,(\mu \text {-a.e. })\right. \text {. }
$$

This statement was proved in [13] (1.7.i), and used in [9] and [14] in various settings. The proofs in the present situation are the same. To understand that this is a weak form of property (Q), namely with respect to $y$ only, and with respect to a given sequence $y_{k}(t), u_{k}(t), k=1,2, \ldots$, we may consider the intermediate definition obtained by replacing the bracketed expression in (7.1) by $\cup_{s=h}^{\infty} \widetilde{Q}\left(t, y_{k_{s}}(t)\right)$. Besides, we are referring here to a property (Q) at only the points $y(t)$ of the trajectory as in Section 2, that is, along the trajectory). The characterizations of Sections 3-5 can be expressed in a weaker form in terms of sequences, and still sufficient to guarantee this weak form of property (Q).

\section{(b) Lipschitz-type criteria}

Here are several specific criteria for property (D). This property implies the weak form of property (Q) stated in (7.1). We have mentioned these criteria in [15] and [9, 13, 14]. They are repeated here in the present context, since they hold not only in Euclidean spaces but also in Banach space situations (cf [14], pp. 446-447, properties $\left(F_{p}\right)$ and $\left(F_{\infty}\right)$ ).

( $\mathrm{Lg}$ ) Lipschitz type conditions, geometric viewpoint $\left(\mathrm{F}_{\mathrm{p}}\right), 1 \leqslant p<\infty$. Here $B$ and $Y$ are Banach spaces, $U$ a metric space. If $1 \leqslant p \leqslant \infty$, let us assume that there are constants $c, \gamma_{,}, \zeta_{0}, p^{\prime}, \quad c \geqslant 0$, $\xi_{0} \geqslant 0, \quad 0 \leqslant \gamma \leqslant p, \quad p^{\prime}=p(p-\gamma)^{-1}$ if $0<\gamma<p, \quad p^{\prime}=\infty$ if $\gamma=p$, and functions $F(t) \geqslant 0$, $t \in G, \quad F \in L_{p^{\prime}}(G, R), \quad h(\zeta), \quad 0 \leqslant \zeta<\infty, \quad h$ monotone nondecreasing, $h(0+)=0, \quad h(\zeta)$ $\leqslant c|\zeta|^{y}$ for $\zeta \geqslant \zeta_{0}$, such that for all $\left(t, y_{1}, u\right),\left(t, y_{2}, u\right) \in S, \quad t \in G-T_{0}$, meas $T_{0}=0$, we have

$$
\left\|\tilde{f}\left(t, y_{1}, u\right)-\tilde{f}\left(t, y_{2}, u\right)\right\|_{B} \leqslant F(t) h\left(\left\|y_{1}-y_{2}\right\|_{Y}\right) \text {. }
$$

Let $\xi(t), y(t), \eta_{k}(t), \bar{\eta}_{k}(t), \xi_{k}(t), \xi_{k}(t), y_{k}(t), u_{k}(t), \phi(t), t \in G, k=1,2, \ldots$, be as in (2.viii); assume that for $\mu$-a.a. $t \in G$ the set $\tilde{Q}(t, y(t))$ is closed and convex, and that $\left\|y_{k}-y\right\|_{p} \rightarrow 0$ as $k \rightarrow \infty$. Then property (Q) holds as in (7.ii) for $\mu$-a.a. $t \in G$, and the lower closure property holds as in (2.viii).

Analogously, we have $\left(F_{\infty}\right)$ : Let $B, Y, U$ as above. Let us assume that there are functions 
$F(t) \geqslant 0, \quad t \in G, \quad F \in L_{1}(G, R)$, such that (7.2) holds. Let $\xi(t), y(t), \eta_{k}(t), \bar{\eta}_{k}(t), \xi_{k}(t), \bar{\xi}_{k}(t), y_{k}(t), u_{k}(t)$, $\phi(t), t \in G, k=1,2, \ldots$, be as in (2.viii); assume that for $\mu$-a.a. $t \in G$ the set $\widetilde{Q}(t, y(t))$ is closed and convex, and that $\left\|y_{k}-y\right\|_{\infty} \rightarrow 0$ as $k \rightarrow \infty$. Then property (Q) holds as in (7.ii) for $\mu$-a.a. $t \in G$, and the lower closure property holds as in (2.viii).

(Lf) Lipschitz type conditions, functional viewpoint: $\left(F_{\mathrm{p}}^{\prime}\right), 1 \leqslant p<\infty$. Let $\{u(t)\}$ be a family of control functions, and let us assume that for every control function $u=u(t)$ of the class there is a function $F_{u}(t)$ with the following properties. First let $p, c, \gamma, \zeta_{0}, p^{\prime}, h$ as in $\left(\mathrm{F}_{\mathrm{p}}\right)$, and let $F_{u}(t) \geqslant 0$, $t \in G, F_{u} \in L_{p}(G, R),\left\|F_{u}\right\|_{p^{\prime}} \leqslant M$, and

$$
\left\|\tilde{f}\left(t, y_{1}, u(t)\right)-\tilde{f}\left(t, y_{2}, u(t)\right)\right\|_{B} \leqslant F_{u}(t) h\left(\left\|y_{1}-y_{2}\right\|_{Y}\right) .
$$

Let $\xi(t), y(t), \eta_{k}(t), \bar{\eta}_{k}(t), \xi_{k}(t), \bar{\xi}_{k}(t), y_{\gamma}(t), u_{k}(t), \phi(t), t \in G, k=1,2, \ldots$, be as in (2.viii) with $u_{k} \in\{u(t)\}$ for all $k$, assume that for $\mu$-a.a. $t \in G$ the set $Q(t, y(t))$ is closed and convex, and that $\left\|y_{k}-y\right\|_{p} \rightarrow 0$ as $k \rightarrow \infty$. Then property (Q) holds as in (7.ii), and the lower closure property holds as in (2.viii).

In particular, $F$ may be simply a function of $(t, u)$, say $F=F(t, u)$, or $F_{u}(t)=F(t, u(t))$, and in this case we need require that $\int_{G}\|F(t, u(t))\|^{p} \mathrm{~d} t \leqslant M$.

Analogously, we have $\left(\mathrm{F}_{\infty}^{\prime}\right)$ : Let $B, Y, U$ as above. Let us assume that there is a function $h(\zeta)$, $0 \leqslant \zeta<+\infty, h$ monotone nondecreasing, $h(0+)=0$, and for every $u \in\{u(t)\}$ a function $F_{u}(t) \geqslant 0, \quad F_{i} \in L_{1}(G, R),\left\|F_{u}\right\|_{1} \leqslant M$, such that (7.3) holds for all $u \in\{u(t)\}$. Let $\xi(t), y(t), n_{k}(t)$, $\bar{\eta}_{k}(t), \xi_{k}(t), \bar{\xi}_{k}(t), y_{k}(t), u_{k}(t), \phi(t), t \in G, k=1,2, \ldots$, be as in (2.viii) with $u_{k} \in\{u(t)\}$ for all $k$, assume that for $\mu$-a.a. $t \in G$ the set $Q(t, y(t))$ is closed and convex, and that $\left\|y_{k}-y\right\|_{\infty} \rightarrow 0$ as $k \rightarrow \infty$. Then property $(Q)$ holds as in (7.ii), and the lower closure property holds as in (2.viii).

In particular, $F$ may be simply a function of $(t, u)$, say $F=F(t, u)$, or $F_{u}(t)=F(t, u, t)$ ), and in this case we need require that $\int_{G} F(t, u(t)) \mathrm{d} t \leqslant M$.

Remark. L. Berkovitz [19] and G. R. Bates [20] state that in their lower closure theorems property (Q) does not hold. They assume a Lipschitz condition $\left|f\left(t, y_{1}, u\right)-f\left(t, y_{2}, u\right)\right| \leqslant F(t, u) \omega\left(\left|y_{1}-y_{2}\right|\right)$ as above and the further assumption $\int_{t_{1}}^{t_{2}} F(t, u(t)) \mathrm{d} t \leqslant A$. From the above it is apparent that property $(Q)$ does hold in their situation. Both Berkovitz and Bates seem not to have realized this in their statements. Their proofs are not much different from ours in the analogous context.

(c) Tempered growth type conditions. Both state and control variables in $L_{p}$ spaces [14].

$(\mathrm{Gg})$ (Tempered growth type conditions). $\left(G_{p q}\right)$ : Here $B, Y, U$ are Banach spaces, the control space $U(t) \subset U$ depends on $t$ only, $1 \leqslant p, q<\infty$, there exist a continuous function $\phi(u)$, or $\phi: U \rightarrow U$, with $\|\phi(u)\|_{U} \rightarrow+\infty$ as $\|u\|_{U} \rightarrow+\infty$, constants $L_{0}, L \geqslant 0, c, c^{\prime} \geqslant 0$, and $\alpha, \beta$, $0<\alpha \leqslant p, \quad 0<\beta \leqslant q$, and a scalar function $\psi(t) \geqslant 0, t \in G, \psi \in L_{1}(G, R)$, such that

$$
\left\|\tilde{f}\left(t, y_{1}, u\right)-\tilde{f}\left(t, y_{2}, u\right)\right\|_{B} \leqslant \psi(t)+c\left(\left\|y_{1}\right\|^{p \alpha}+\left\|y_{2}\right\|^{p-x}\right)+c^{\prime}\|\phi(u)\|_{U}^{q-\beta} \text {. }
$$

Let $\xi(t), y(t), \eta_{k}(t), \bar{\eta}_{k}(t), \xi_{k}(t), \xi_{k}(t), y_{k}(t), u_{k}(t), \phi(t), t \in G, k=1,2, \ldots$, be as in (2.viii) with $y, y_{k} \in$ $L_{p}(G, Y), u_{k}$ measurable, $\phi \bigcirc u_{k} \in L_{q}(G, U),\|y\|\left\|_{p},\right\| y_{k}\left\|_{p} \leqslant L_{0},\right\| \phi\left(u_{k}\right) \|_{q} \leqslant L, y_{k}(t) \rightarrow y(t)$ in measure in $G$ as $k \rightarrow \infty$. Then, property (Q) holds as in (7.ii) for $\mu$-a.a. $t \in G$, and the lower closure property holds as in (2.viii).

The conditions $\left(\mathrm{G}_{\infty q}\right),\left(\mathrm{G}_{p \infty}\right),\left(\mathrm{G}_{\infty \infty}\right)$ can be expressed analogously.

For instance $\left(\mathrm{G}_{\infty q}\right): 1 \leqslant q<\infty$, there exist a continuous function $\phi(u)$, or $\phi: U \rightarrow U$, with $\|\phi(u)\|_{U} \rightarrow+\infty$ as $\|u\|_{U} \rightarrow+\infty$, constants $L_{0}, L \geqslant 0, c^{\prime} \geqslant 0, \beta, 0<\beta \leqslant q$, a scalar function $\psi(t) \geqslant 0, t \in G, \psi \in L_{1}(G, R)$, and a monotone nondecreasing function $\sigma(\zeta) \geqslant 0,0 \leqslant \zeta<+\infty$, 
such that

$$
\| \tilde{f}\left(t, y_{1}, u\right)-\tilde{f}\left(t, y_{2}, u\left\|_{B} \leqslant \psi(t) \sigma\left(\left\|y_{1}\right\|+\left\|y_{2}\right\|\right)+c^{\prime}\right\| \phi(u) \|_{U}^{q-\beta} .\right.
$$

Note that we do not assume $\sigma(0+)=0$. The conditions $\left(\mathrm{G}_{p q}\right),\left(\mathrm{G}_{\infty q}\right)$ are only growth conditions. We leave it to the reader to state the conditions $\left(G_{p \infty}\right)$ and $\left(G_{\infty \infty}\right)$ analogous to the ones above.

(Gf) (Tempered growth type conditions, functional viewpoint). Let $\{u(t)\}$ be a family of control functions, and let us assume that for every control function $u=u(t)$ of the class there are functions $W(t), V(t), t \in G$, which may depend on $u(t)$ (we may denote them as $W_{u}(t), V_{u}(t)$ ), and constants $c, c^{\prime}, \alpha, \beta$ as above, such that the functions $W \in L_{1}(G, R)$ are equiabsolutely integrable on $G$, $V \in L_{q}(G, U)$ with $\|V\|_{q} \leqslant M$ for some constant $M$, and

$$
\left\|f\left(t, y_{1}, u(t)\right)-f\left(t, y_{2}, u(t)\right)\right\|_{B} \leqslant W(t)+c\left(\left\|y_{1}\right\|^{p-\alpha}+\left\|y_{2}\right\|^{p-\alpha}\right)+\|V(t)\|_{q}^{q-\beta} .
$$

Let $\xi(t), y(t), \eta_{k}(t), \bar{\eta}_{k}(t), \xi_{k}(t), \bar{\xi}_{k}(t), y_{k}(t), u_{k}(t), \phi(t), t \in G, k=1,2, \ldots$, be as in (2.viii) with $y, y_{k} \in$ $L_{p}(G, Y), u_{k}$ measurable, $V_{u_{k}}(t) \in L_{q}(G, U),\|y\|_{p},\left\|y_{k}\right\|_{p} \leqslant L_{0},\left\|V_{u_{k}}\right\|_{q} \leqslant L, y_{k}(t) \rightarrow y(t)$ in measure in $G$ as $k \rightarrow \infty$. Then the same conclusions hold as above.

It is enough to know that for each $k$ there are functions $W_{k}(t), V_{k}(t), t \in G$, as before.

We leave it to the reader to state the analogous conditions $\left.\left(G_{\infty}\right),\left(G_{p \infty}\right), G_{\infty, \infty}\right)$.

(d) Tempered growth type conditions. Only the control variables in $L_{q}[14]$.

$\left(\mathrm{Hg}\right.$ ) (tempered growth type conditions $H$, geometric view point). For $1 \leqslant q<\infty, y, y_{k}$ measurable, $y_{k}(t) \rightarrow y(t)$ in measure in $G$ as $k \rightarrow+\infty, u_{k} \in L_{q}(G, U),\left\|u_{k}\right\|_{q} \leqslant L$, a constant, and there are other constants $c^{\prime}, \beta, 0<\beta \leqslant q$, and a scalar function $\psi(t) \geqslant 0, t \in G, \psi \in L_{1}(G, R)$, such that

$$
\left\|f\left(t, y_{1}, u\right)-f\left(t, y_{2}, u\right)\right\|_{B} \leqslant \psi(t)+c^{\prime}\left\|u^{q-\beta}\right\| .
$$

For $q=\infty$, the condition above becomes $\left(H_{x}\right): y, y_{k}$ are measurable, $y_{k}(t) \rightarrow y(t)$ in measure in $G$ as $k \rightarrow \infty, u_{k} \in L_{\infty}(G, U)$, and there are a function $\psi(t), t \in G, \psi \in L_{1}(G, R)$, and a monotone nondecreasing function $\sigma(\zeta) \geqslant 0,0 \leqslant \zeta<+\infty$, such that

$$
\left\|f\left(t, y_{1}, u\right)-f\left(t, y_{2}, u\right)\right\|_{B} \leqslant \psi(t) \sigma\left(\|u\|_{U}\right) .
$$

\section{REFERENCES}

1. CeSARI L., Existence theorems for weak and usual optimal solutions in Lagrange problems with unilateral constraints, I and II. Trans. Amer. Math. Soc. 124, 369-412, 413-430 (1966).

2. CeSari L., Existence theorems of optimal control of the Mayer type, SIAM J. Control 6, 517-552 (1968).

3. Goodman G. S., The duality of convex functions and Cesari's property (Q) J. Optim. Theory Applic. 19, 17-23 (1976). Also: Proc. Conference Zakopane (1974).

4. IOFFE A. D., On lower semicontinuity of integral functionals, SIAM J. Control 15, 521-538 (1977).

5. Olech C., Existence theorems for optimal problems with vector valued cost functions. Trans. Amer. Math. Soc. 136, 157-180 (1969).

6. Oцeсн C., Existence theory in optimal control problems. The underlying ideas, International Conference on Differential Equations (Edited by H. A. Antosiewicz) pp. 612-629. Academic Press (1975).

7. RockAFELlaR R. T., Integral functionals, normal integrands and measurable selection, Nonlinear Operators and the Calculus of Variations (Edited by J. P. Gossez et al.), Springer Lecture Notes Math. 543, 157-207 (1976).

8. Cesari L. \& Suryanarayana M. B., Existence theorems for Pareto optimization. Multivalued and Banach space valued functionals. Trans. Amer. Math. Soc. 244, 37-65 (1978); An existence theorem for Pareto problems, Nonlinear Analysis TMA 2, 225-233 (1978).

9. Cesari L. \& Suryanarayana M. B., Nemitsky's operators and lower closure theorems. J. Optim. Theory Applic. 19, 165-183 (1976). 
10. CeSARI L., Seminormality and upper semicontinuity in optimal control, J. Optim. Theory Applic. 6, 114-137 (1970).

11. Cesari L., Closure, lower closure and semicontinuity theorems in optimal control, SIAM J. Control 9, 287-315 (1971).

12. CESARI L., Closure theorems for orientor fields and weak convergence, Archive ration. Mech. Analysis 55, $332-356$ (1974).

13. Cesari L. \& Suryanarayana M. B., Convexity and property (Q) in optimal control theory, SIAM J. Control 12, 705-720 (1974).

14. Cesari L. \& Suryanarayana M. B., Closure theorems without seminormality conditions, J. Optim. Theory Applic. 15, 441-465 (1975).

15. CeSARI L., Geometric and analytic views in existence theorems for optimal control in Banach spaces. I, II, III. J. Optim. Theory Applic. 14, 505-520 (1974); 15, 467-497 (1975); 19, 185-214 (1976).

16. Kaiser P. J. \& Suryanarayana M. B., Orientor field equations in Banach spaces, J. Optim. Theory Applic. 19, 141-164 (1976).

17. Cesari L., Problems of Optimization. Springer-Verlag (1979).

18. C.astaing C. \& Valadier M., Convex Analysis and Measurable Multifunctions, Lecture notes in Mathematics, no. 580, Springer Verlag, New York (1977).

19. Berkovitz L., Optimal Control Theory, pp. 98-99. Springer (1974).

20. Bates G. R., Lower closure and existence theorems for optimal control problems with infinite horizon. $J$. Optim. Theory Applic. 24, 639-649 (1978).

21. CeSARi L., A necessary and sufficient condition for lower semicontinuity. Bull. Amer. Math. Soc. 80, 467-472 (1974).

22. SURyanARAYANA M. B., Upper semicontinuity of set valued functions. To appear.

23. Denkowski $Z$., The convergence of generalized sequences of sets and functions in the locally convex spaces. Preprint 151, Inst. of Math., Polish Acad. of Sciences, Warsaw 1978. 